\title{
Structural Color Spectral Response of Dense Structures of Discoidal Particles Generated by Evaporative Assembly
}

Tianyu Liu ${ }^{\dagger}$, Tianyu Liu ${ }^{\ddagger}$, Fengyi Gao ${ }^{\ddagger}$, Sharon C. Glotzer ${ }^{\ddagger}$, and Michael J. Solomon ${ }^{\dagger s^{*}}$

${ }^{\dagger}$ Macromolecular Science and Engineering, University of Michigan, Ann Arbor, Michigan, 48109, United States

Chemical Engineering, University of Michigan, Ann Arbor, Michigan 48109, United States

šBiointerfaces Institute, University of Michigan, Ann Arbor, Michigan 48109, United States

*Email:mjsolo@umich.edu

\begin{tabular}{cccc}
\hline Initial sphere diameter $(\mathrm{nm})$ & Discoid a $(\mathrm{nm})$ & Discoid c $(\mathrm{nm})$ & Discoid aspect ratio \\
\hline $215 \pm 3$ & $252 \pm 2$ & $156 \pm 2$ & $0.62 \pm 0.01$ \\
$258 \pm 6$ & $296 \pm 4$ & $196 \pm 2$ & $0.66 \pm 0.01$ \\
$286 \pm 8$ & $324 \pm 10$ & $224 \pm 6$ & $0.69 \pm 0.03$ \\
$286 \pm 8$ & $362 \pm 8$ & $180 \pm 4$ & $0.50 \pm 0.02$ \\
$286 \pm 8$ & $420 \pm 6$ & $134 \pm 2$ & $0.32 \pm 0.01$ \\
$358 \pm 10$ & $410 \pm 8$ & $274 \pm 4$ & $0.67 \pm 0.02$ \\
\hline
\end{tabular}

Table S1. The initial sphere sizes, the length of the major axes (a), minor axes (c), and aspect ratios of the discoid particles used in the experiment are reported. 


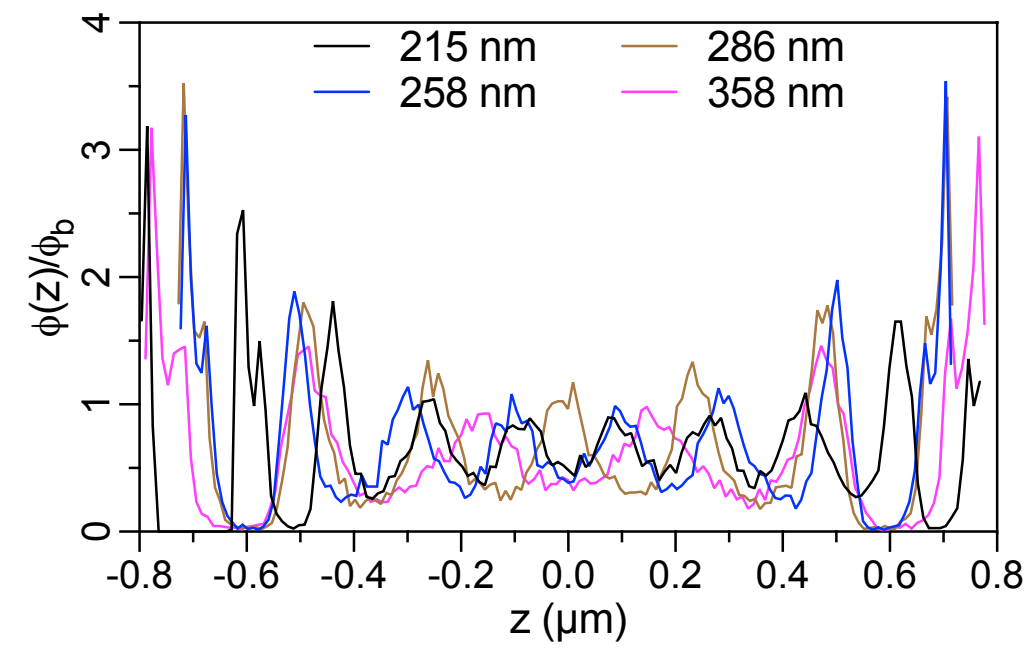

Figure S1. Normalized Z-density distributions of discoid packings from different sized particles confined between two walls. 